
\title{
28 Research Suare \\ Identifying and Ranking Potential Driver Genes of Alzheimer's Disease Using Weighted Co-Expression Network Analysis
}

\section{Liang-Yong Xia}

Xi'an Jiaotong University

\section{Lihong Tang}

Xi'an Jiaotong University

\section{Hui Huang}

Shanghai Jiao Tong University

Jie Luo ( $\nabla$ jieluo@sjtu.edu.cn )

Shanghai Jiao Tong University

\section{Research}

Keywords: Alzheimer's disease, Genetic variation, Transcriptomic, Differentially expressed genes, Weighted gene co-expression network analysis

Posted Date: June 7th, 2021

DOI: https://doi.org/10.21203/rs.3.rs-529357/v1

License: (c) (i) This work is licensed under a Creative Commons Attribution 4.0 International License. Read Full License 


\title{
RESEARCH
}

\section{Identifying and ranking potential driver genes of Alzheimer's disease using weighted co-expression network analysis}

\author{
Liang-Yong Xia ${ }^{1,2}$, Lihong Tang ${ }^{1}$, Hui Huang ${ }^{1}$ and Jie Luo ${ }^{1 *}$
}

\author{
${ }^{*}$ Correspondence: \\ jieluo@sjtu.edu.cn \\ ${ }^{1}$ School of Biomedical \\ Engineering,Shanghai Jiao Tong \\ University, 800 Dongchuan Road, \\ 200030 Shanghai, China \\ Full list of author information is \\ available at the end of the article
}

\begin{abstract}
Background: Alzheimer's disease (AD) is one of the most common neurodegenerative diseases. Identification of AD-related genes from transcriptomics provided new direction to the mechanism for finding potential targets for drug therapy.

Methods: We mined gene co-expression network modules from differentially expressed genes (DEGs) of $A D$ and normal samples in multiple datasets by weighted gene co-expression network analysis (WGCNA). A convergent functional genomic (CFG) method was used to prioritize potential driver genes.

Results: The 7567 DEGs were enriched significantly with 61 KEGG pathway and $242 \mathrm{GO}$ terms. Then, the genes in $5 \mathrm{AD}$-specific modules obtained significantly from DEGs were interconnected with well-known AD risk genes in common PPI network. Remarkably, compared to the number of Tau production-related genes, $A \beta$ play a more critical role. Lastly, the 23 potential driver genes was prioritized by CFG method from 5 AD-specific modules.
\end{abstract}

Conclusions: Identification of AD-related genes could be useful for understanding pathophysiology of $A D$ and looking for candidates drug targets.

Keywords: Alzheimer's disease; Genetic variation; Transcriptomic; Differentially expressed genes; Weighted gene co-expression network analysis

\section{Background}

Alzheimer's disease (AD) is one of the most common neurodegenerative diseases, accounting for the majority of dementia patients[1]. AD is estimated to affect in 13.8 million individuals in the United States (US), with 7.0 million being aged 85 years or older by $2050[2,3]$. Currently, genetic factor are believed to be partially responsible for $\mathrm{AD}[4]$. Genome-wide association studies (GWAS) have also revealed that some single nucleotide polymorphisms (SNPs) contribute to AD disease onset $[5,6]$. These include common variants such as amyloid protein precursor (APP), presenilin-1 (PSEN1), presenilin-2 (PSEN2) and apolipoprotein E (APOE). PSEN1, PSEN2 and APP genes are clear pathogenic genes of early-onset $\mathrm{AD}[7]$. APOE, as the only identified risk gene for late-onset $\mathrm{AD}$, can increase the rate of cognitive decline[8]. Different microRNAs (miRNAs) are also involved in the pathophysiology of $\mathrm{AD}$ [9]. For example, miRNA-377 promotes cell proliferation and inhibits cell apoptosis by regulating the expression level of cadherin 13 (CDH13), thus participating in the occurrence and development of $\mathrm{AD}[10]$. Long non-coding RNAs (lncRNAs) have 
been widely reported to be associated with a variety of physiological and pathological processes, such as AD. Brain cytoplasmic RNA is a kind of lncRNA, and the overexpression of brain cytoplasmic may lead to synaptic/dendritic degeneration in $\mathrm{AD}[11]$. Despite the fact that remarkable advances have been made in the understanding of the genetic basis of $\mathrm{AD}$, there is no pharmaceutical or other intervention that can treat $\mathrm{AD}$. Identification of $\mathrm{AD}$-related genes from transcriptomics becomes an attractive strategy for finding potential targets for drug therapy.

Gene expression profiling of transcriptomic datasets of $\mathrm{AD}$ and normal brain samples has identified potential genes and contributed to the search for potential targets[12]. Recently, most studies on AD transcriptome analysis pay attention to the identification of DEGs from different brain regionsand there are only few work studied highly correlated pairs of genes to obtain gain or loss of co-expression in $\mathrm{AD}$ [13]. Generally, describing the degree of association between two genes can be obtained by calculating correlation coefficients such as Pearson and Spearman between expression values. But the disadvantage of a fixed threshold approach is that the threshold is artificially defined and it ignores a lot of potential correlations. At the same time, this approach can also lose information about the trend of genetic changes, and it will be difficult to describe the strength of correlation in the network. In order to solve these problems, the idea of weighting is proposed. WGCNA is widely used to describe the association pattern between gene expression in microarrays or RNA-seq, and can be divided the gene co-expression network of complex biological processes into several highly related feature modules. The feature modules represent several groups of highly coordinated gene sets, associate the modules with specific clinical features to find genes that perform key functions, and help identify potential mechanisms involved in specific biological processes and explore candidate biomarkers[14, 15].

In this paper, we aimed to identify potential driver genes for AD from DEGs using WGCNA and CFG methods based on multiple transcriptomics dataset. Firstly, the datasets was download from AlzData and ADNI database. Then genes with $|\log F C|>0.1$ and $P<0.05$ were regarded as DEGs. We also identified AD-specific modules using WGCNA method. Lastly, we prioritized potential driver genes by the CFG method in Figure 1.

\section{Materials and Methods}

\section{AD expression data collection and preprocessing}

All the original microarray data regarding $\mathrm{AD}$ were downloaded from AlzData(http://www.alzdata.org/)and Alzheimer's Disease Neuroimaging Initiative(http://adni.loni.usc.edu/). We have 467 controls and 309 AD from five dataset for subsequent analyses, including EC (39 vs 39$), \operatorname{HP}(67$ vs 74$), \mathrm{FC}(128$ vs 104), TC(39 vs 52) and ADNI(194 vs 40). Only ADNI had $A \beta$ and Tau related data. Detailed information of each dataset is shown in Table-1. Of note, to solve

data imbalanced in ADNI dataset, we randomly chose 40 samples from the control in 10 times.

\section{AD data detection of DEGs and enrichment of biological process}

In order to identify potential genes of $\mathrm{AD}$, differential expression analysis was conducted by $\mathrm{R}$ package edgeR and the Benjamini-Hochberg's method was used to 
correct for multiple comparisons[16]. For each dataset, t-test was used to identify DEGs with a cut-off of statistical significance $P<0.05$ and foldchange $>0.1$. Functional enrichment of the DEGs was produced from Database for DAVID, which now provides a comprehensive set of functional annotation tools for investigators to understand biological meaning behind large list of genes. For obtained list of DEGs, DAVID is able to identify enriched biological themes, particularly KEGG pathway and GO terms.

\section{Weighted gene co-expression network analysis}

We use WGCNA approach, which is good for distinguishing the strong and weak relationships, to divide the gene co-expression network of complex biological processes into several highly related feature modules. For genes $\mathrm{i}$ and $\mathrm{j}$, the correlation coefficient is $r_{i j}$, we define the correlation intensity $: a_{i j}=r_{i j}^{\beta}$, which depends on the choice of power $\beta$. The $\beta$ value is determined by the lowest value close to the scale-free network.

According to this idea, the correlation strength of expression levels among all genes was calculated, and the adjacency matrix was obtained. Further, the topological overlap matrix (TOM) was calculated. In simple terms, if genes $\mathrm{i}$ and $\mathrm{j}$ have a lot of the same adjacent genes, then $T O M_{i, j}$ will be high, which means that there are similar expression patterns between the two genes. Therefore, several AD-specific modules from DEGs of AD and normal samples could be obtained by WGCNA.

\section{Protein-protein interaction(PPI) network}

Mentioned AD-specific modules before, to identify the genes in these AD-specific modules and the functional relationship between well-known AD risk genes(APP, PSEN1, PSEN2, APOE or MAPT), a protein-protein interaction(PPI) network was constructed by Gephi(https://gephi.org/).

\section{Convergent functional genomics}

The potential driver genes was prioritized from $\mathrm{AD}$-specific modules by $\mathrm{CFG}$ method, which integrated various levels of AD-related evidence [4, 17]. There were five AD-related evidence: (1) regulation of target gene expression by $\mathrm{AD}$ genetic variants;(2) association at least one locus significantly ;(3) physical interaction between target genes and APP, PSEN1, PSEN2, APOE or MAPT;(4) differential expression of target gene before AD pathology emergence;(5) correlation of target gene expression with $\mathrm{AD}$ pathology in $A \beta$ and Tau. The range of CFG score was from 0 to 5 , with 5 indicating highest priority.

\section{Results DEG detection}

A total of 776 samples and 108,302 genes from multiple transcriptomic datasets were compiled for DEGs detection $(P<0.05$ and foldchange $>0.1)$. Besides, for ADNI dataset, we randomly chose 40 samples from the control in 10 times and selected gene with frequency greater than or equal to 3. Each red node represented DEG for five datasets(Figure 2). We identified 7567 DEG(2166 EC, 1952 HP, 949 FC, 3075 
TC and $3204 \mathrm{ADNI}$ ) for subsequent analyses. About 6\% $19 \%$ of the total genes could be identified as DEGs. Among the DEG list in all five datasets, the expression patterns of well-known AD risk genes, such as APP, PSEN1, PSEN2, APOE and MAPT were only slightly altered or unchanged in AD patients. In addition, 19 genes had a consistently differential expression from EC, HP, FC, TC and ADNI(Figure 3).

\section{Functional annotation of AD-related DEGs}

We investigated functional enrichment of the AD-related DEGs. The 7567 target genes in the network were enriched in 324 KEGG pathway and 1381 GO terms in Figure 4. We identified 61 KEGG pathway and 324 GO terms $(P<0.005)$, respectively. As shown in Table-2, we also found several pathways have been reported to be associated with AD, including Alzheimer disease pathway, MAPK signaling pathway and AMPK signaling pathway. Top 20 significantly KEGG pathway selected was exhibited for each dataset in Figure 5. Besides, these GO terms are divided into ontologies based on a hierarchical relations. Specifically, DEGs related to the biological processes for synaptic-related functions were significant enriched in Table-3, such as chemical synaptic transmission, regulation of postsynaptic membrane potential, synaptic vesicle exocytosis, synaptic transmission, GABAergic, regulation of synaptic transmission, glutamatergic, synaptic vesicle endocytosis and long-term synaptic potentiation. In addition, they were associated with neuron-related processes, including neurotransmitter secretion, neuron projection morphogenesis, negative regulation of neuron apoptotic process and negative regulation of neuron projection development. These results of KEGG pathway and GO terms could contribute to understand the neuropathological process of AD.

\section{DEG-enriched modules}

We used WGCNA to divide the DEGs into several highly related gene modules. As shown in Figure 6, a very significant positive correlation was observed between five modules and $\mathrm{AD}$ for five dataset. A modular size was ranged from 96 to 142 genes that might reflect the different layers and complexity of gene regulation in the $\mathrm{AD}$ brain. These five DEG-enriched modules were used for identifying potential driver genes for AD etiology and pathology. We obtained a PPI network from five ADspecific modules, which contained 5 seed genes and 209 neighbors. Due to the lack of connectivity, several genes were not included in the PPI network, such as S100A6, PSAT1 and MXI1. Notably, only a few genes were linked to all five well-known AD risk genes (APP, PSEN1, PSEN2, APOE and MAPT) in Figure 7.

\section{Pathology correlation $A \beta$ and Tau}

We also investigated the relationship between the genes in these five AD-specific modules and AD pathology in $A \beta$ and Tau. Table- 4 shows that there were significant differences in AD pathology among different datasets. Compared with the number of Tau production-related genes, AD pathology in $A \beta$ play a vital role. These results could contributed to understanding the pathology process of AD. 


\section{Potential driver genes of AD}

To search potential driver genes of $\mathrm{AD}$, we identified 23 related-AD genes based on CFG score $(>=4)$ and $\mathrm{P}$-value $(<0.05)$ from the genes in five $\mathrm{AD}$-specific module. We also list previously published gene-based studies for the identification of patients with AD in Table-5. For example, GJA1, also known as connexin 43, shows upregulated mRNA and protein levels in $\mathrm{AD}[18]$.

\section{Discussion}

In this study, we aimed to identify potential driver genes for $\mathrm{AD}$ from multiple transcriptomics dataset DEGs by WGCNA and CFG methods.

Pathway enrichment analysis was performed to interpret the function of these DEGs. KEGG pathway analysis for the 7567 DEGs were significantly enriched in one KEGG pathway "MAPK signaling pathway", which is composed of ERK, P38, and JNK. In the adult nervous system, ERK activation is necessary for synaptic plasticity and memory formation[19]. In the brains of AD patients, P38 is highly expressed. $A \beta$-induced P38 activation increases tau phosphorylation and promotes the amyloidogenic processing of $\mathrm{APP}[20,21]$. In a mouse model of $\mathrm{AD}$, the JNK signaling pathway is overactivated in the spine before cognitive decline[22]. These studies indicate that the overactivation of MAPK signaling pathway could cause the occurrence of $\mathrm{AD}$. Therefore, preventing MAPK overactivation is effective strategy in order to reduce $A \beta$ deposition, Tau hyperphosphorylation, neuronal apoptosis, and memory impairment. MAPKs could be potential targets for novel and effective therapeutics of $\mathrm{AD}[23,24]$.

GO term analysis indicated that the 7567 DEGs were mainly involved in chemical synaptic transmission, regulation of postsynaptic membrane potential, synaptic vesicle exocytosis, synaptic transmission, GABAergic synapses, regulation of synaptic transmission, glutamatergic, synaptic vesicle endocytosis, long-term synaptic potentiation, neurotransmitter secretion, neuron projection morphogenesis, negative regulation of neuron apoptotic process and negative regulation of neuron projection development. Damage to neuronal and synaptic function has always been considered an important pathological feature of neurodegenerative diseases, and decreased synaptic activity is also considered to be the most relevant pathological feature of $\mathrm{AD}$ cognitive impairment[25]. For example, the downregulation of GABAergic synapses is closely related to the loss of GABAergic inhibition[26]. Studies have found that GABAergic neurotransmission is closely related to various aspects of AD pathology, including $A \beta$ toxicity and Tau hyperphosphorylation[27]. The level of GABA inhibitory neurotransmitter in $\mathrm{AD}$ patients was significantly reduced, suggesting that $\mathrm{AD}$ has insufficient synaptic function and neuronal transmission[28]. In addition, In a mouse model of $\mathrm{AD}$ indicate that the impairment of hippocampal neurogenesis may be mediated by GABAergic signal dysfunction or the imbalance between excitatory and inhibitory synapses[29]. Therefore, GABAergic synapses not only plays an important role in the function of the hippocampus, but also in the pathogenesis of $\mathrm{AD}$.

To explore the protein interactions, a PPI network was consisted of 5 well-known AD risk genes(APP, PSEN1, PSEN2, APOE and MAPT) and 209 genes. The 14 genes with greater than four degrees (including CXCL12,BAX, IRF2, IRF5, SMARCC1, SP1, CASP6, DCN, FZD2, GNA13, HNF4A, ITGAV, PELP1 and SDC2) 
were selected. Especilally, CXCL12 plays a major role in neuroinflammation because it mediates the local immune response and attracts leukocytes, which are thought to migrate across the blood-brain barrier along the concentration gradient of chemokines to their targets. This occurs for example in AD in the vicinity of the amyloid plaques that attract and/or activate local glial cells[30].

\section{Limitations}

There are some limitations in this study. First, although we identified 23 potential driver genes of AD by the WGCNA and CFG method, these approachs could be used to prioritize genes rather than to identify true causal genes. Therefore, further biological validation of the identified genes are necessary in future studies. Second, 4 of 5 datasets were downloaded from AlzData, which only retained the common genes from different studies during the cross-platform normalization. Third, the sample size of EC, HP and TC available for analyze was still limited, and the larger sample size of FC and ADNI might have a greater influence on the results. Fourth, the rapid development of various omics provide new opportunities for understanding of AD. However, we only used transcriptomics dataset to identify potential driver genes of AD. Finally, more potential genes of AD were not considered. Deep learning has capacity to dig out more hidden gene in data and is a machine learning algorithm based on artificial neural network, which is a computational model inspired by the structure of human brain. The main difference between deep learning and traditional artificial neural network lies in the scale and complexity of network structure. The networks of deep learning have a larger number of hidden layers, while traditional artificial neural networks usually have only one hidden layer. This is due to the lack of big data and GPU hardware technical support in the last century. Due to the emergence of more powerful CPU and GPU hardware, deep learning with more hidden layers is proposed on the basis of artificial neural network, and more nodes can be used in each hidden layer[31, 32].

\section{Conclusions}

In this study, we identified potential driver genes from AD-specific modules using multiple transcriptomics datasets and observed that DEGs were enriched with several pathways significantly, which are consistent with observations from previous studies. In summary, Our results contribute to understanding pathophysiology of $\mathrm{AD}$ and looking for candidates drug targets.

\section{List of abbreviations}

AD: Alzheimer's disease ; DANI: Alzheimer's Disease Neuroimaging Initiative; EC: Entorhinal Cortex; HP: Hippocampus; TC: Temporal Cortex; FC: Frontal Cortex; DEGs: Differentially expressed genes; WGCNA: Weighted gene coexpression network analysis; DAVID: Database for Annotation, Visualization and Integrated Discovery; KEGG: Kyoto Encyclopedia of Genes and Genomes; GO: Gene Ontology; $A \beta$ : $\beta$-amyloid; GWAS: Genome-wide association studies; SNP: Single nucleotide polymorphisms; GCN: Gene co-expression network ; CFG: Convergent functional genomic 


\title{
Declarations
}

\author{
Ethics approval and consent to participate
}

Not applicable

Consent for publication

Not applicable

Availability of data and materials

AlzData and ADNI data are publicly available.

Competing interests

The authors declare that they have no competing interests.

Funding

This work is supported by Postdoctoral Research Foundation of China[2020M671125].

\section{Author's contributions}

L.Y.X., and L.L.T. contributed to collect data sets and analyze data. L.Y.X., L.L.T., H.H., and J.L. contributed to the interpretation of the results. L.Y.X. took the lead in writing the manuscript. L.Y.X., L.L.T., H.H., and J.L.

revised the manuscript.

\section{Acknowledgements}

Data used in the preparation of this article were obtained from the from AlzData(http://www.alzdata.org/) and Alzheimer's Disease Neuroimaging Initiative (ADNI) database (adni.loni.usc.edu).

\section{Author details}

${ }^{1}$ School of Biomedical Engineering,Shanghai Jiao Tong University, 800 Dongchuan Road, 200030 Shanghai, China. ${ }^{2}$ Faculty of Information Technology, Macau University of Science and Technology., Avenida Wai Long, Taipa, 999078 Macau, China.

\section{References}

1. Darby, R.R., Joutsa, J., Fox, M.D.: Network localization of heterogeneous neuroimaging findings. Brain 142(1), 70-79 (2019)

2. Association, A., et al.: 2018 alzheimer's disease facts and figures. Alzheimer's \& Dementia 14(3), 367-429 (2018)

3. Cummings, J., Lee, G., Ritter, A., Sabbagh, M., Zhong, K.: Alzheimer's disease drug development pipeline: 2019. Alzheimer's \& Dementia: Translational Research \& Clinical Interventions 5, 272-293 (2019)

4. Xu, M., Zhang, D.-F., Luo, R., Wu, Y., Zhou, H., Kong, L.-L., Bi, R., Yao, Y.-G.: A systematic integrated analysis of brain expression profiles reveals yap1 and other prioritized hub genes as important upstream regulators in alzheimer's disease. Alzheimer's \& Dementia 14(2), 215-229 (2018)

5. Hao, S., Wang, R., Zhang, Y., Zhan, H.: Prediction of alzheimer's disease-associated genes by integration of gwas summary data and expression data. Frontiers in genetics 9, 653 (2019)

6. Andrews, S.J., Fulton-Howard, B., Goate, A.: Interpretation of risk loci from genome-wide association studies of alzheimer's disease. The Lancet Neurology 19(4), 326-335 (2020)

7. Lanoiselée, H.-M., Nicolas, G., Wallon, D., Rovelet-Lecrux, A., Lacour, M., Rousseau, S., Richard, A.-C., Pasquier, F., Rollin-Sillaire, A., Martinaud, O., et al.: App, psen1, and psen2 mutations in early-onset alzheimer disease: A genetic screening study of familial and sporadic cases. PLoS medicine 14(3), 1002270 (2017)

8. Wijsman, E.M., Pankratz, N.D., Choi, Y., Rothstein, J.H., Faber, K.M., Cheng, R., Lee, J.H., Bird, T.D., Bennett, D.A., Diaz-Arrastia, R., et al.: Genome-wide association of familial late-onset alzheimer's disease replicates bin1 and clu and nominates cugbp2 in interaction with apoe. PLoS Genet 7(2), 1001308 (2011)

9. Femminella, G.D., Ferrara, N., Rengo, G.: The emerging role of micrornas in alzheimer's disease. Frontiers in physiology 6, 40 (2015)

10. Liu, F., Zhang, Z., Chen, W., Gu, H., Yan, Q.: Regulatory mechanism of microrna-377 on cdh13 expression in the cell model of alzheimer's disease. Eur. Rev. Med. Pharmacol. Sci 22, 2801-2808 (2018)

11. Doxtater, K., Tripathi, M.K., Khan, M.M.: Recent advances on the role of long non-coding rnas in alzheimer's disease. Neural Regeneration Research 15(12), 2253 (2020)

12. Patel, H., Dobson, R.J., Newhouse, S.J.: A meta-analysis of alzheimer's disease brain transcriptomic data. Journal of Alzheimer's Disease 68(4), 1635-1656 (2019)

13. Xiang, S., Huang, Z., Wang, T., Han, Z., Christina, Y.Y., Ni, D., Huang, K., Zhang, J.: Condition-specific gene co-expression network mining identifies key pathways and regulators in the brain tissue of alzheimer's disease patients. BMC medical genomics 11(6), 39-51 (2018)

14. Nangraj, A.S., Selvaraj, G., Kaliamurthi, S., Kaushik, A.C., Cho, W.C., Wei, D.Q.: Integrated ppi-and wgcna-retrieval of hub gene signatures shared between barrett's esophagus and esophageal adenocarcinoma. Frontiers in pharmacology 11, 881 (2020)

15. Ren, Z.-H., Shang, G.-P., Wu, K., Hu, C.-Y., Ji, T.: Wgcna co-expression network analysis reveals ilf3-as1 functions as a cerna to regulate ptbp1 expression by sponging mir-29a in gastric cancer. Frontiers in genetics 11, 39 (2020) 
16. Hackstadt, A.J., Hess, A.M.: Filtering for increased power for microarray data analysis. BMC bioinformatics 10(1), 1-12 (2009)

17. Ayalew, M., Le-Niculescu, H., Levey, D., Jain, N., Changala, B., Patel, S., Winiger, E., Breier, A., Shekhar, A., Amdur, R., et al.: Convergent functional genomics of schizophrenia: from comprehensive understanding to genetic risk prediction. Molecular psychiatry 17(9), 887-905 (2012)

18. Ren, R., Zhang, L., Wang, M.: Specific deletion connexin43 in astrocyte ameliorates cognitive dysfunction in app/ps1 mice. Life sciences 208, 175-191 (2018)

19. Du, Y., Du, Y., Zhang, Y., Huang, Z., Fu, M., Li, J., Pang, Y., Lei, P., Wang, Y.T., Song, W., et al.: Mkp-1 reduces a $\beta$ generation and alleviates cognitive impairments in alzheimer's disease models. Signal transduction and targeted therapy 4(1), 1-12 (2019)

20. Gourmaud, S., Paquet, C., Dumurgier, J., Pace, C., Bouras, C., Gray, F., Laplanche, J.-L., Meurs, E.F., Mouton-Liger, F., Hugon, J.: Increased levels of cerebrospinal fluid jnk3 associated with amyloid pathology: links to cognitive decline. Journal of psychiatry \& neuroscience: JPN 40(3), 151 (2015)

21. Giraldo, E., Lloret, A., Fuchsberger, T., Viña, J.: A $\beta$ and tau toxicities in alzheimer's are linked via oxidative stress-induced p38 activation: protective role of vitamin e. Redox biology 2, 873-877 (2014)

22. Sclip, A., Tozzi, A., Abaza, A., Cardinetti, D., Colombo, I., Calabresi, P., Salmona, M., Welker, E., Borsello, T.: c-jun n-terminal kinase has a key role in alzheimer disease synaptic dysfunction in vivo. Cell death \& disease 5(1), 1019-1019 (2014)

23. Feld, M., Krawczyk, M.C., Sol Fustinana, M., Blake, M.G., Baratti, C.M., Romano, A., Boccia, M.M.: Decrease of erk/mapk overactivation in prefrontal cortex reverses early memory deficit in a mouse model of alzheimer's disease. Journal of Alzheimer's Disease 40(1), 69-82 (2014)

24. Yenki, P., Khodagholi, F., Shaerzadeh, F.: Inhibition of phosphorylation of jnk suppresses a $\beta$-induced er stress and upregulates prosurvival mitochondrial proteins in rat hippocampus. Journal of Molecular Neuroscience 49(2), 262-269 (2013)

25. Wu, M., Fang, K., Wang, W., Lin, W., Guo, L., Wang, J.: Identification of key genes and pathways for alzheimer's disease via combined analysis of genome-wide expression profiling in the hippocampus. Biophysics Reports 5(2), 98-109 (2019)

26. Kim, S., Kim, H., Park, D., Kim, J., Hong, J., Kim, J.S., Jung, H., Kim, D., Cheong, E., Ko, J., et al.: Loss of iqsec3 disrupts gabaergic synapse maintenance and decreases somatostatin expression in the hippocampus. Cell reports 30(6), 1995-2005 (2020)

27. Kadoyama, K., Matsuura, K., Takano, M., Otani, M., Tomiyama, T., Mori, H., Matsuyama, S.: Proteomic analysis involved with synaptic plasticity improvement by gabaa receptor blockade in hippocampus of a mouse model of alzheimer's disease. Neuroscience research 165, 61-68 (2021)

28. Schmitz, T.W., Correia, M.M., Ferreira, C.S., Prescot, A.P., Anderson, M.C.: Hippocampal gaba enables inhibitory control over unwanted thoughts. Nature communications 8(1), 1-12 (2017)

29. Sun, B., Halabisky, B., Zhou, Y., Palop, J.J., Yu, G., Mucke, L., Gan, L.: Imbalance between gabaergic and glutamatergic transmission impairs adult neurogenesis in an animal model of alzheimer's disease. Cell stem cell 5(6), 624-633 (2009)

30. Guyon, A.: Cxcl12 chemokine and its receptors as major players in the interactions between immune and nervous systems. Frontiers in cellular neuroscience 8, 65 (2014)

31. Esteva, A., Robicquet, A., Ramsundar, B., Kuleshov, V., DePristo, M., Chou, K., Cui, C., Corrado, G., Thrun, S., Dean, J.: A guide to deep learning in healthcare. Nature medicine 25(1), 24-29 (2019)

32. Zou, J., Huss, M., Abid, A., Mohammadi, P., Torkamani, A., Telenti, A.: A primer on deep learning in genomics. Nature genetics 51(1), 12-18 (2019)

33. Tan, M.G., Lee, C., Lee, J.H., Francis, P.T., Williams, R.J., Ramírez, M.J., Chen, C.P., Wong, P.T.-H., Lai, M.K.: Decreased rabphilin 3a immunoreactivity in alzheimer's disease is associated with a $\beta$ burden. Neurochemistry international 64, 29-36 (2014)

34. Flores, J., Noël, A., Foveau, B., Lynham, J., Lecrux, C., LeBlanc, A.C.: Caspase-1 inhibition alleviates cognitive impairment and neuropathology in an alzheimer's disease mouse model. Nature communications 9(1), 1-14 (2018)

35. Emilsson, L., Saetre, P., Jazin, E.: Low mrna levels of rgs4 splice variants in alzheimer's disease: association between a rare haplotype and decreased mrna expression. Synapse 59(3), 173-176 (2006)

36. Xiao, M.-F., Xu, D., Craig, M.T., Pelkey, K.A., Chien, C.-C., Shi, Y., Zhang, J., Resnick, S., Pletnikova, O., Salmon, D., et al.: Nptx2 and cognitive dysfunction in alzheimer's disease. Elife 6, 23798 (2017)

37. Feldheim, J., Kessler, A.F., Schmitt, D., Salvador, E., Monoranu, C.M., Feldheim, J.J., Ernestus, R.-I., Löhr, M., Hagemann, C.: Ribosomal protein s27/metallopanstimulin-1 (rps27) in glioma-a new disease biomarker? Cancers 12(5), 1085 (2020)

38. Fujita, Y., Maeda, T., Sato, C., Sato, M., Hatakeyama, H., Ota, Y., Iwabuchi, N., Tatesawa, K., Nomura, A., Zou, K., et al.: Engulfment of toxic amyloid $\beta$-protein in neurons and astrocytes mediated by megf10. Neuroscience 443, 1-7 (2020)

39. Lan, Y.-L., Zhao, J., Ma, T., Li, S.: The potential roles of aquaporin 4 in alzheimer's disease. Molecular neurobiology 53(8), 5300-5309 (2016)

40. Conti, E., Andreoni, S., Tomaselli, D., Storti, B., Brovelli, F., Acampora, R., Da Re, F., Appollonio, I., Ferrarese, C., Tremolizzo, L.: Serum dbi and biomarkers of neuroinflammation in alzheimer's disease and delirium. Neurological Sciences, 1-5 (2020)

41. Li, T., Zhu, J.: Entanglement of ccr5 and alzheimer's disease. Frontiers in aging neuroscience 11, 209 (2019)

42. Salter, M.W., Stevens, B.: Microglia emerge as central players in brain disease. Nature medicine 23(9), 1018 (2017)

43. Wu, Q., Kong, W., Wang, S.: Peripheral blood biomarkers cxcl12 and tnfrsf13c associate with cerebrospinal fluid biomarkers and infiltrating immune cells in alzheimer disease. Journal of Molecular Neuroscience, 1-10 (2021)

44. Kaczmarczyk, L., Reichenbach, N., Blank, N., Jonson, M., Dittrich, L., Petzold, G., Jackson, W.S.: Insights 
into calcium signaling and gene expression in astrocytes uncovered with 129s4 slc1a3-2a-creert2 knock-in mice. bioRxiv (2020)

45. Gertje, E.C., Janelidze, S., van Westen, D., Palmqvist, S., Hansson, O., Mattsson, N.: White matter lesions are associated with csf biomarkers of neuroinflammation in prodromal alzheimer's disease: Biomarkers

(non-neuroimaging)/novel biomarkers. Alzheimer's \& Dementia 16, 041795 (2020)

Figures

Figure 1 A flowchart of the whole study. (1) Data collection from AlzData and ADNI; (2) Data preprocessing; (3)DEGs regarded with $|\log F C|>0.1$ and $P<0.05$; (4)Enrichment of biological process analyzed by DAVID; (5) Use WGCNA to find AD-specific module; (6) Generate a complete list from AD-specific module; (7) CFG based on AD-related evidence to prioritize driver genes of $A D$.

Figure 2 Enhanced Volcano for illustrating DEGs in all datasets. The red node represents DEGs with $|\log F C|>0.1$ and $P<0.05$. (A) EC,(B) HP,(C) FC,(D) TC and (E) ADNI. Note:in ADNI dataset, DEGs by counting the frequency of 3 or above out of 10 occurrences.

Figure 3 Venn diagram for DEGs. Venn diagram is used to represent relationships between EC (blue),HP (red), FC (green), TC (yellow) and ADNI (brown).

Figure 4 Venn diagram for KEGG pathway and GO term. venn diagram is used to represent relationships between multiple datasets. (A) KEGG pathway and (B) GO term

Figure 5 Top 20 pathway of KEGG for five datasets (P-value<0.005). (A) EC, (B) HP, (C) FC, (D) TC and (E) ADNI

Figure 6 Module-trait relationships for five datasets. Each row represents different gene co-expression modules, and each column represents different clinical phenotypes. Number represent correlation coefficients and $\mathrm{P}$-values are in parenthesis. Correlation strength is represented by continuous color, with red being positive, blue being negative. (A) EC, (B) HP, (C) FC, (D) TC and (E) ADNI

Figure 7 PPI network. The PPI network contained 5 well-known AD risk genes (green) and 209 (yellow) neighbors, which are from five AD-specific modules.

Tables 
Table 1 Brief Descriptions for five datasets. These datasets come from AlzData and ADNI, respectively. Each dataset has multiple features. SDs are given in parentheses.

\begin{tabular}{|c|c|c|c|c|c|}
\hline \multirow{2}{*}{ Dataset } & \multicolumn{4}{|c|}{ AlzData } & \multirow{2}{*}{ Alzheimer's Disease Neuroimaging Initiative } \\
\hline & Entorhinal Cortex & Hippocampus & Frontal Cortex & Temporal Cortex & \\
\hline Abbreviation & EC & $\mathrm{HP}$ & FC & TC & ADNI \\
\hline No.of.gene & 15361 & 16313 & 11779 & 15462 & 49387 \\
\hline Sample size(Control/AD) & $78(39 / 39)$ & $141(67 / 74)$ & $232(128 / 104)$ & $91(39 / 52)$ & $234(194 / 40)$ \\
\hline Age & $80(29.6)$ & $81.7(9.6)$ & $83(9.4)$ & $81(8.7)$ & $74.3(6.5)$ \\
\hline Male/Female/Unknown & $35 / 43 / 0$ & $68 / 73 / 0$ & $99 / 111 / 22$ & $32 / 41 / 18$ & $116 / 118 / 0$ \\
\hline$A \beta$ & NA & NA & NA & NA & $1142.9(494.9)$ \\
\hline Tau & NA & NA & NA & NA & $25.4(11.6)$ \\
\hline
\end{tabular}

Table 2 Significant KEGG pathways obtained from DAVID (P-value $<0.005)$. The first column is KEGG pathway ID; the second column is the name of KEGG pathway.

\begin{tabular}{|c|c|c|c|}
\hline ID & Description & ID & Description \\
\hline hsa00020 & Citrate cycle (TCA cycle) & hsa04966 & Collecting duct acid secretion \\
\hline hsa00190 & Oxidative phosphorylation & hsa05010 & Alzheimer disease \\
\hline hsa00260 & $\begin{array}{l}\text { Glycine, serine and threonine } \\
\text { metabolism }\end{array}$ & hsa05012 & Parkinson disease \\
\hline hsa00620 & Pyruvate metabolism & hsa05014 & Amyotrophic lateral sclerosis \\
\hline hsa01200 & Carbon metabolism & hsa05016 & Huntington disease \\
\hline hsa01210 & 2-Oxocarboxylic acid metabolism & hsa05017 & Spinocerebellar ataxia \\
\hline hsa01230 & Biosynthesis of amino acids & hsa05020 & Prion disease \\
\hline hsa01522 & Endocrine resistance & hsa05022 & $\begin{array}{l}\text { Pathways of neurodegeneration - } \\
\text { multiple diseases }\end{array}$ \\
\hline hsa03050 & Proteasome & hsa05032 & Morphine addiction \\
\hline hsa04010 & MAPK signaling pathway & hsa05033 & Nicotine addiction \\
\hline hsa04070 & Phosphatidylinositol signaling system & hsa05110 & Vibrio cholerae infection \\
\hline hsa04071 & Sphingolipid signaling pathway & hsa05120 & $\begin{array}{l}\text { Epithelial cell signaling in Heli- } \\
\text { cobacter pylori infection }\end{array}$ \\
\hline hsa04110 & Cell cycle & hsa05131 & Shigellosis \\
\hline hsa04120 & Ubiquitin mediated proteolysis & hsa05132 & Salmonella infection \\
\hline hsa04137 & Mitophagy - animal & hsa05140 & Leishmaniasis \\
\hline hsa04140 & Autophagy - animal & hsa05145 & Toxoplasmosis \\
\hline hsa04144 & Endocytosis & hsa05152 & Tuberculosis \\
\hline hsa04145 & Phagosome & hsa05163 & Human cytomegalovirus infection \\
\hline hsa04152 & AMPK signaling pathway & hsa05167 & $\begin{array}{l}\text { Kaposi sarcoma-associated her- } \\
\text { pesvirus infection }\end{array}$ \\
\hline hsa04211 & Longevity regulating pathway & hsa05169 & Epstein-Barr virus infection \\
\hline hsa04218 & Cellular senescence & hsa05202 & $\begin{array}{l}\text { Transcriptional misregulation in } \\
\text { cancer }\end{array}$ \\
\hline hsa04260 & Cardiac muscle contraction & hsa05205 & Proteoglycans in cancer \\
\hline hsa04360 & Axon guidance & hsa05212 & Pancreatic cancer \\
\hline hsa04625 & $\begin{array}{l}\text { C-type lectin receptor signaling path- } \\
\text { way }\end{array}$ & hsa05214 & Glioma \\
\hline hsa04666 & Fc gamma R-mediated phagocytosis & hsa05215 & Prostate cancer \\
\hline hsa04721 & Synaptic vesicle cycle & hsa05219 & Bladder cancer \\
\hline hsa04722 & Neurotrophin signaling pathway & hsa05220 & Chronic myeloid leukemia \\
\hline hsa04723 & $\begin{array}{l}\text { Retrograde endocannabinoid signal- } \\
\text { ing }\end{array}$ & hsa05223 & Non-small cell lung cancer \\
\hline hsa04920 & Adipocytokine signaling pathway & hsa05225 & Hepatocellular carcinoma \\
\hline hsa04932 & Non-alcoholic fatty liver disease & hsa05235 & $\begin{array}{l}\text { PD-L1 expression and PD-1 } \\
\text { checkpoint pathway in cancer }\end{array}$ \\
\hline hsa04961 & $\begin{array}{l}\text { Endocrine and other factor-regulated } \\
\text { calcium reabsorption }\end{array}$ & & \\
\hline
\end{tabular}


Table 3 Significant GO terms obtained from DAVID ( $P$-value $<0.005)$. The first column is GO terms ID; the second column is the name of GO terms.

\begin{tabular}{|c|c|}
\hline ID & Term \\
\hline GO:0002223 & stimulatory C-type lectin receptor signaling pathway \\
\hline GO:0006888 & ER to Golgi vesicle-mediated transport \\
\hline GO:0048015 & phosphatidylinositol-mediated signaling \\
\hline GO:0038128 & ERBB2 signaling pathway \\
\hline GO:0007249 & I-kappaB kinase/NF-kappaB signaling \\
\hline GO:0006672 & ceramide metabolic process \\
\hline GO:0000165 & MAPK cascade \\
\hline GO:0045944 & positive regulation of transcription from RNA polymerase II promoter \\
\hline GO:0007269 & neurotransmitter secretion \\
\hline GO:0035329 & hippo signaling \\
\hline GO:0006120 & mitochondrial electron transport, NADH to ubiquinone \\
\hline GO:0042776 & mitochondrial ATP synthesis coupled proton transport \\
\hline GO:0070125 & mitochondrial translational elongation \\
\hline GO:0032981 & mitochondrial respiratory chain complex I assembly \\
\hline GO:0007409 & axonogenesis \\
\hline GO:0048812 & neuron projection morphogenesis \\
\hline GO:0043524 & negative regulation of neuron apoptotic process \\
\hline GO:0007268 & chemical synaptic transmission \\
\hline GO:0060078 & regulation of postsynaptic membrane potential \\
\hline GO:0016079 & synaptic vesicle exocytosis \\
\hline GO:0048813 & dendrite morphogenesis \\
\hline GO:0090263 & positive regulation of canonical Wnt signaling pathway \\
\hline GO:0009967 & positive regulation of signal transduction \\
\hline GO:0051932 & synaptic transmission, GABAergic \\
\hline GO:0046034 & ATP metabolic process \\
\hline GO:0070933 & histone $\mathrm{H} 4$ deacetylation \\
\hline GO:0007420 & brain development \\
\hline GO:0007417 & central nervous system development \\
\hline GO:0035357 & peroxisome proliferator activated receptor signaling pathway \\
\hline GO:0015986 & ATP synthesis coupled proton transport \\
\hline GO:0040029 & regulation of gene expression, epigenetic \\
\hline GO:0007399 & nervous system development \\
\hline GO:0051966 & regulation of synaptic transmission, glutamatergic \\
\hline GO:0048488 & synaptic vesicle endocytosis \\
\hline GO:0010977 & negative regulation of neuron projection development \\
\hline GO:0060071 & Wnt signaling pathway, planar cell polarity pathway \\
\hline GO:0006521 & regulation of cellular amino acid metabolic process \\
\hline GO:2000310 & regulation of $\mathrm{N}$-methyl-D-aspartate selective glutamate receptor activity \\
\hline GO:0038061 & NIK/NF-kappaB signaling \\
\hline GO:0035418 & protein localization to synapse \\
\hline GO:0060291 & long-term synaptic potentiation \\
\hline
\end{tabular}


Table 4 The number of gene of AD pathology in $A \beta$ and Tau are significant for all datasets.

\begin{tabular}{ccccc}
\hline Dataset & $A \beta$ & Tau & $A \beta$ and Tau & In total \\
\hline EC & 21 & 12 & 7 & 40 \\
\hline HP & 8 & 7 & 6 & 21 \\
\hline FC & 24 & 10 & 25 & 59 \\
\hline TC & 50 & 4 & 22 & 76 \\
\hline ADNI & 20 & 14 & 14 & 48 \\
\hline In total & 123 & 47 & 74 & 244 \\
\hline
\end{tabular}

Table 5 The brief description of 23 potential driver genes of AD. The potential 23 driver genes are prioritized by the CFG method, which is integrated various levels of AD-related data including expression of target gene is regulated by eQLT, GWAS, PPI, Early_DEG, Pathology correlation $A \beta$ and Tau. The last column is list previously published gene-based studies for the identification of patients with AD. (*: P-value $<0.05 ;{ }^{* *}$ : P-value $<0.01 ; * * *$ : $\mathrm{P}$-value $\left.<0.001\right)$

\begin{tabular}{|c|c|c|c|c|c|c|c|c|}
\hline \multirow{3}{*}{ Gene } & \multicolumn{6}{|c|}{ AD-related evidence } & \multirow{3}{*}{ CFG } & \multirow{3}{*}{ Reference } \\
\hline & \multirow{2}{*}{ eQTL } & \multirow{2}{*}{ GWAS } & \multirow{2}{*}{ PPI } & \multirow{2}{*}{ Early_DEG } & \multicolumn{2}{|c|}{ Pathology } & & \\
\hline & & & & & $A \beta$ & Tau & & \\
\hline GJA1 & 2 & 2 & PSEN1,MAPT,APOE & yes & $0.388, * *$ & $0.131, \mathrm{~ns}$ & 5 & [18] \\
\hline RPH3A & 5 & 2 & - & yes & $-0.199, \mathrm{~ns}$ & $-0.738, * *$ & 4 & [33] \\
\hline CASP6 & 5 & 0 & $\begin{array}{c}\text { APP,PSEN1,PSEN2, } \\
\text { MAPT }\end{array}$ & yes & $0.482, * * *$ & $0.738, * *$ & 4 & [34] \\
\hline RGS4 & 1 & 32 & - & yes & $-0.419, * *$ & $-0.579, *$ & 4 & [35] \\
\hline NPTX2 & 1 & 1 & - & yes & $-0.688, * * *$ & $-0.783, * * *$ & 4 & [36] \\
\hline RPS27 & 1 & 0 & PSEN2 & yes & $0.503, * * *$ & $0.662, * *$ & 4 & [37] \\
\hline MEGF10 & 3 & 8 & - & yes & $0.559, * * *$ & $0.120, \mathrm{~ns}$ & 4 & [38] \\
\hline AQP4 & 7 & 4 & - & yes & $0.800, * * *$ & $0.275, \mathrm{~ns}$ & 4 & [39] \\
\hline MYT1L & 3 & 12 & - & yes & $-0.488, * * *$ & $-0.583, *$ & 4 & NA \\
\hline CITED2 & 1 & 0 & APP,PSEN1,APOE & yes & $-0.433, * *$ & $-0.772, * * *$ & 4 & NA \\
\hline $\mathrm{CDH} 7$ & 0 & 1 & PSEN1 & yes & $-0.345, *$ & $-0.691, * *$ & 4 & NA \\
\hline DBI & 1 & 1 & - & yes & $0.780, * * *$ & $0.718, * *$ & 4 & [40] \\
\hline PELI2 & 2 & 0 & PSEN2 & yes & $0.591, * * *$ & $-0.107, \mathrm{~ns}$ & 4 & NA \\
\hline AVEN & 1 & 1 & - & yes & $0.525, * * *$ & $0.008, \mathrm{~ns}$ & 4 & NA \\
\hline SLA & 1 & 0 & PSEN1,MAPT & yes & $0.114, \mathrm{~ns}$ & $0.662, * *$ & 4 & NA \\
\hline DCN & 8 & 0 & $\begin{array}{l}\text { APP,PSEN1,MAPT, } \\
\text { APOE }\end{array}$ & yes & $-0.416, * *$ & $0.546, *$ & 4 & NA \\
\hline CCR5 & 1 & 0 & APP & yes & $0.769, * * *$ & $0.616, *$ & 4 & [41] \\
\hline IRF5 & 1 & 0 & $\begin{array}{l}\text { APP,PSEN1,PSEN2, } \\
\text { MAPT,APOE }\end{array}$ & yes & $0.879, * * *$ & $0.839, * * *$ & 4 & [42] \\
\hline CXCL12 & 1 & 0 & $\begin{array}{l}\text { APP,PSEN2,MAPT, } \\
\text { APOE }\end{array}$ & yes & $0.432,{ }^{* *}$ & $-0.069, \mathrm{~ns}$ & 4 & [43] \\
\hline CREM & 1 & 0 & PSEN1,MAPT,APOE & yes & $-0.439, * *$ & $-0.396, \mathrm{~ns}$ & 4 & NA \\
\hline EHHADH & 14 & 0 & MAPT,APOE & yes & $0.438, * *$ & $-0.022, \mathrm{~ns}$ & 4 & NA \\
\hline SLC1A3 & 7 & 1 & - & yes & $0.651, * * *$ & $0.494, \mathrm{~ns}$ & 4 & [44] \\
\hline IL15 & 2 & 18 & - & yes & $0.623, * * *$ & $0.685, * *$ & 4 & [45] \\
\hline
\end{tabular}




\section{Figures}

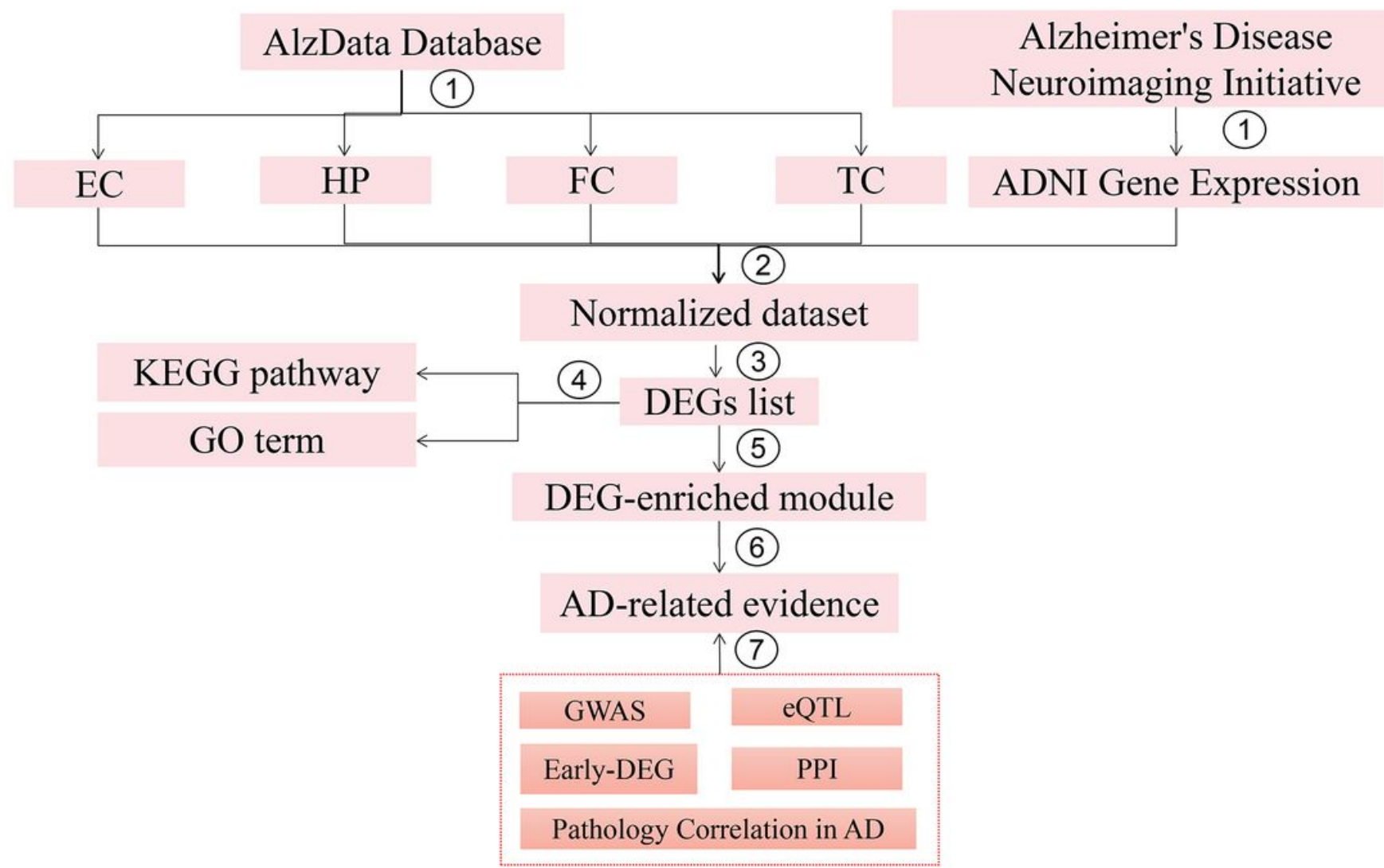

Figure 1

"Please see the Manuscript PDF file for the complete figure caption". 


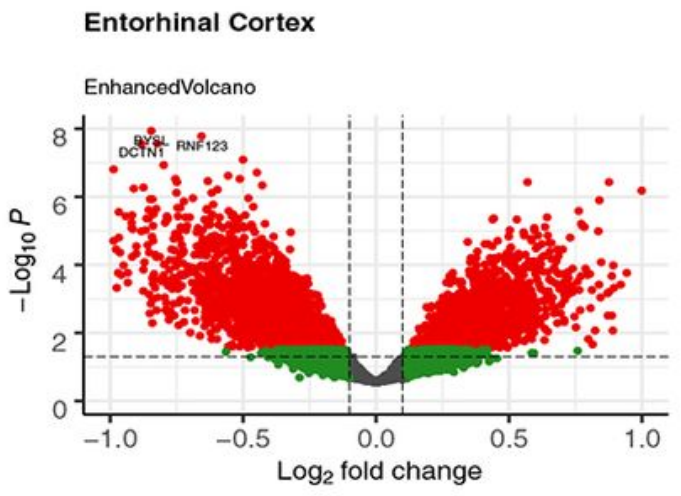

A

- NS - $\log 2 \mathrm{FC}$ - P-valuo $\& \log 2 \mathrm{FC}$

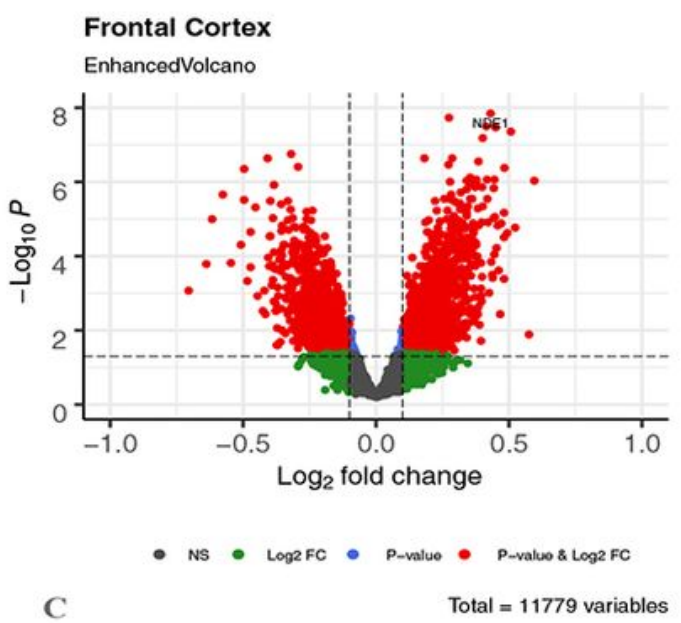

Hippocampus

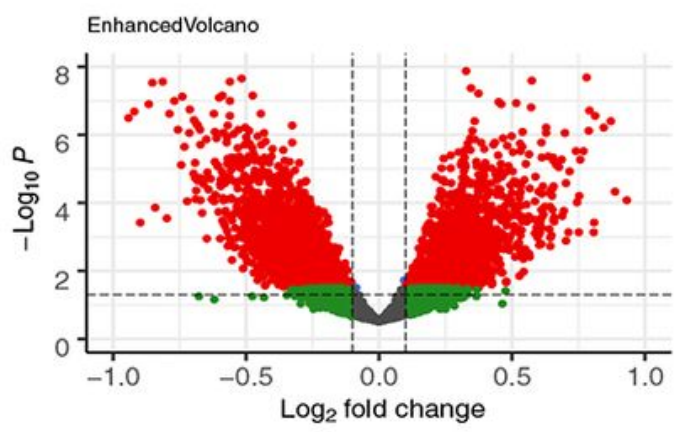

- NS - $\log 2 \mathrm{FC}$ - P-value - P-value \& Log2 FC

B

Total $=16313$ variables

\section{Temporal Cortex}

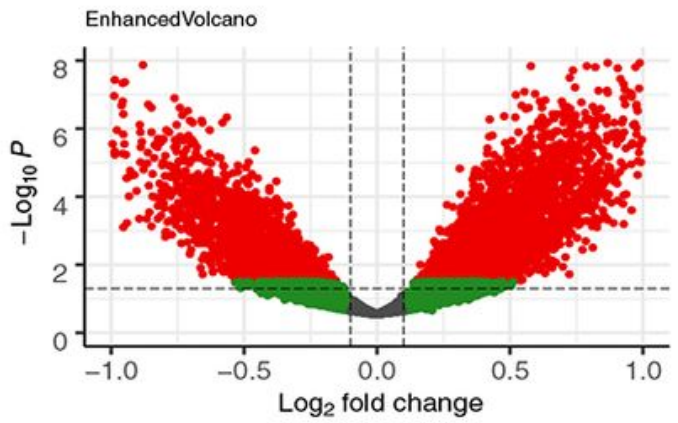

D

Total $=15462$ variables

ADNI(Frequency greater than 3)

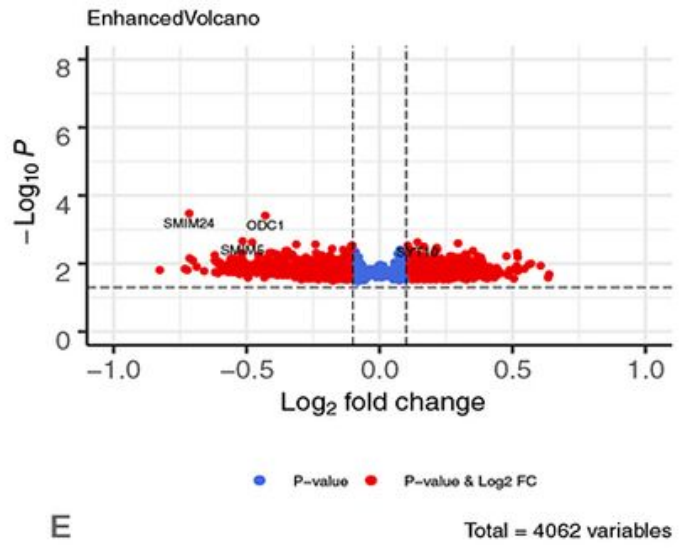

Figure 2

"Please see the Manuscript PDF file for the complete figure caption". 


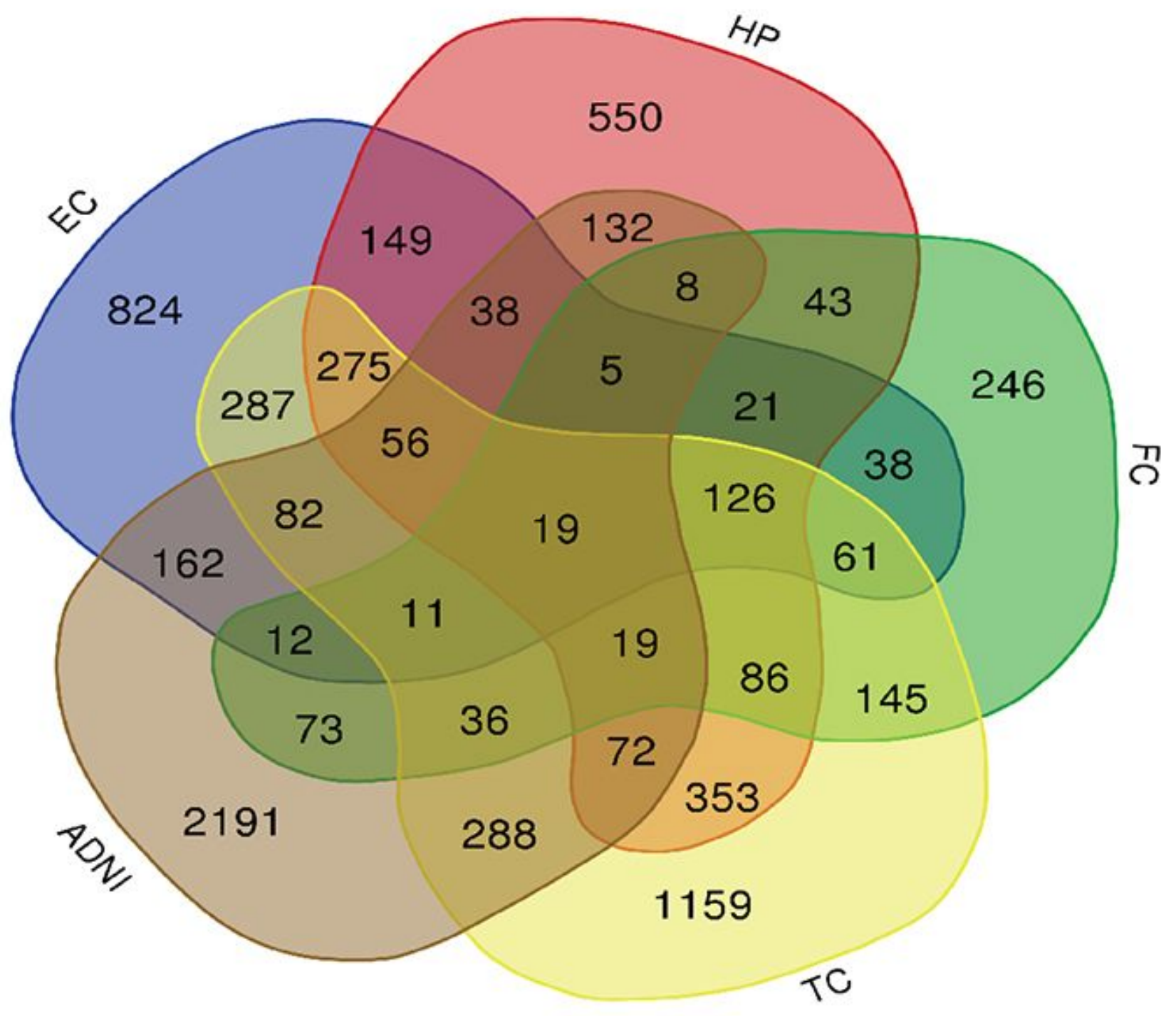

Figure 3

Venn diagram for DEGs. Venn diagram is used to represent relationships between EC (blue),HP (red), FC (green), TC (yellow) and ADNI (brown). 

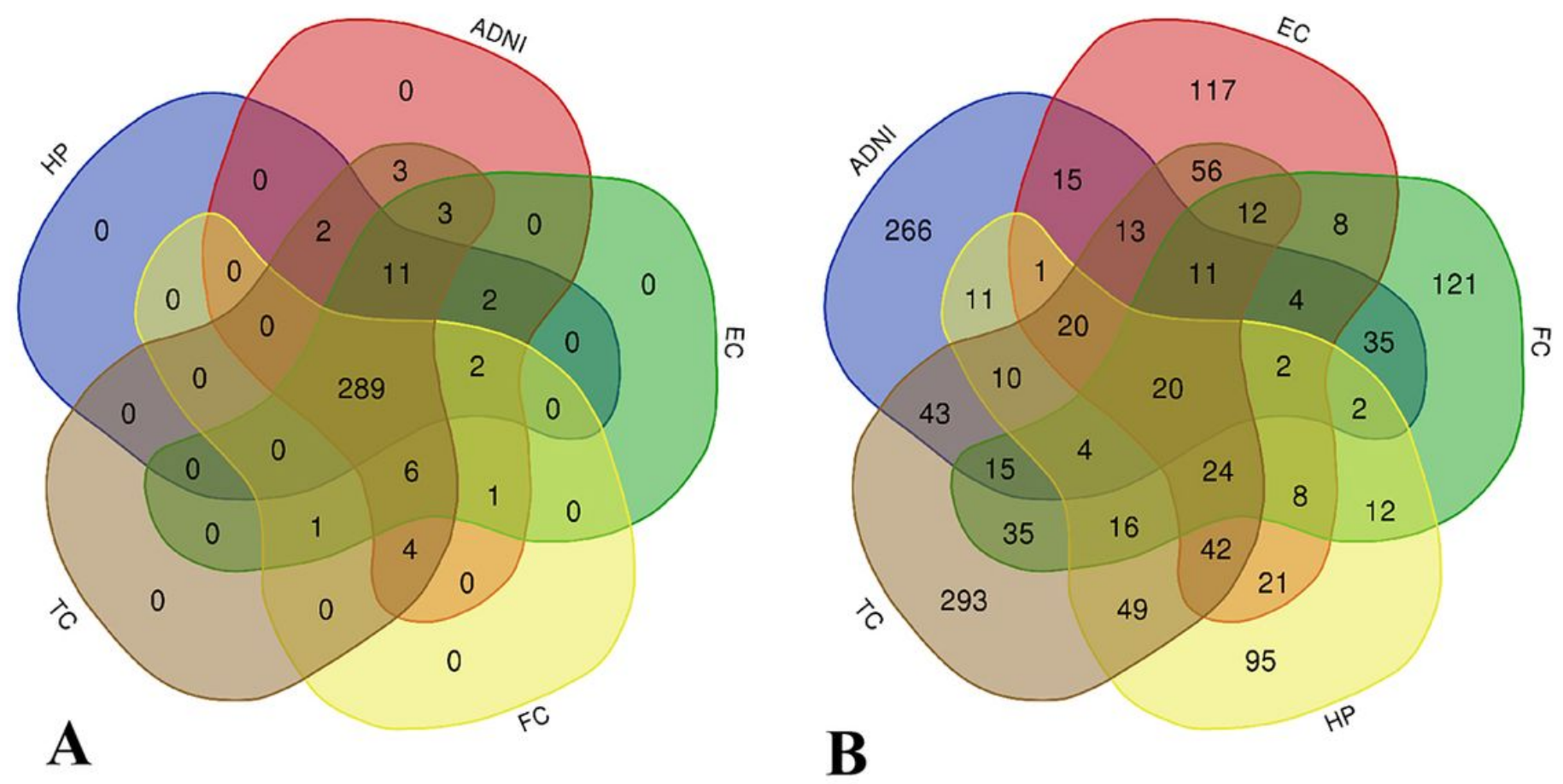

\section{Figure 4}

Venn diagram for KEGG pathway and GO term. venn diagram is used to represent relationships between multiple datasets. (A) KEGG pathway and (B) GO term 

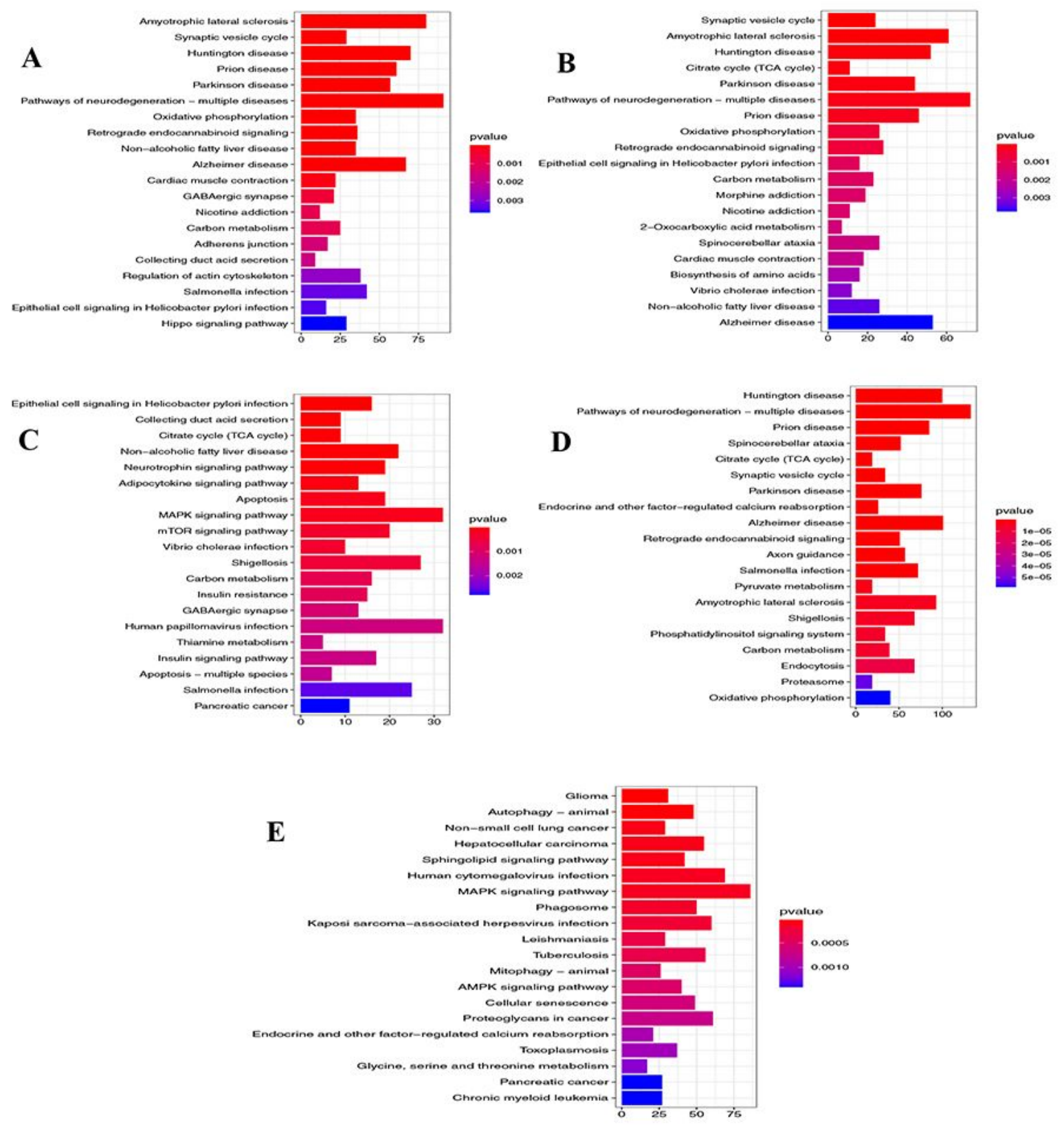

Figure 5

Top 20 pathway of KEGG for five datasets (P-value<0.005). (A) EC, (B) HP, (C) FC, (D) TC and (E) ADNI 

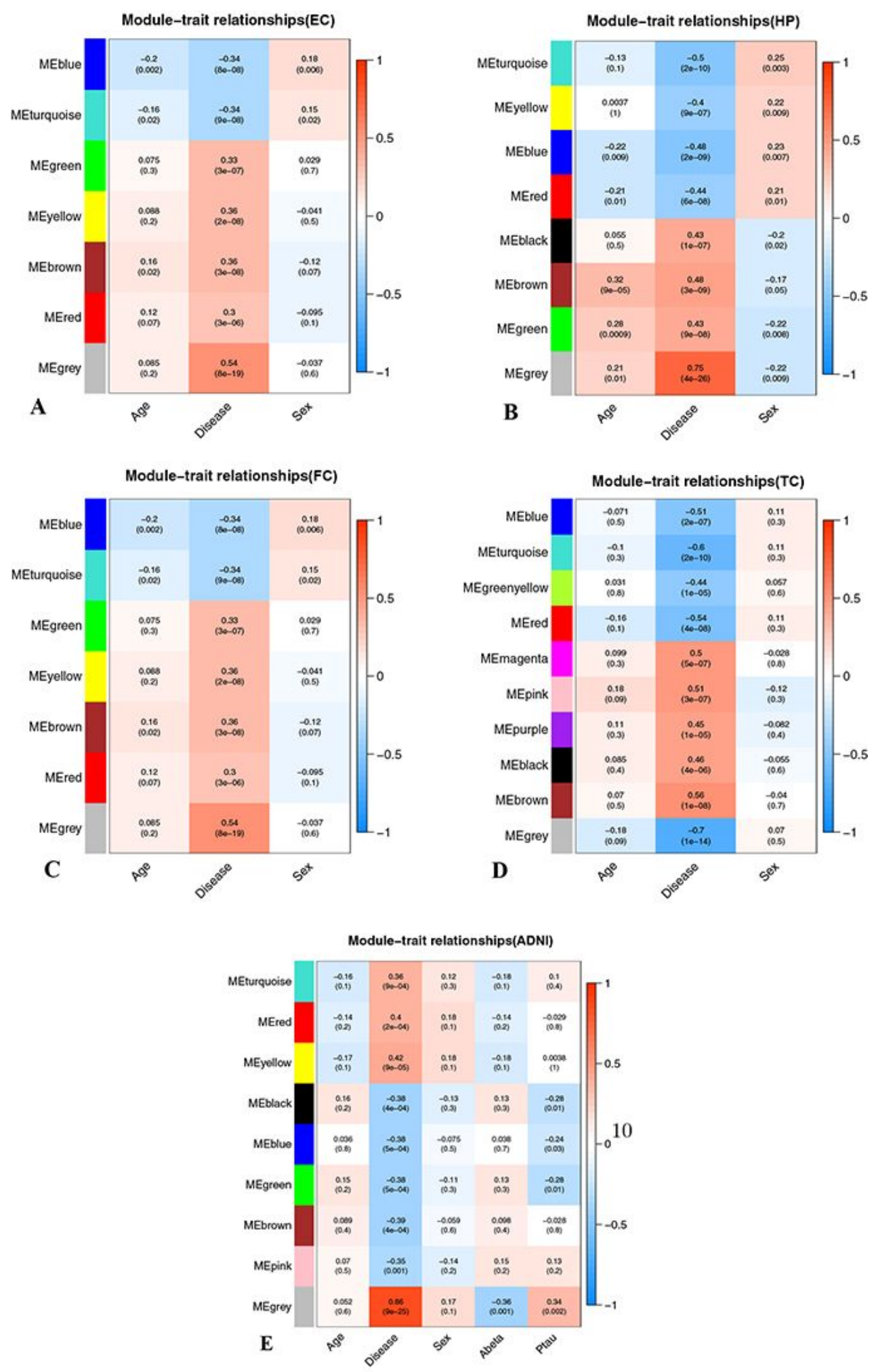

\section{Figure 6}

Module-trait relationships for five datasets. Each row represents different gene co-expression modules, and each column represents different clinical phenotypes. Number represent correlation coeficients and P-values are in parenthesis. Correlation strength is represented by continuous color, with red being positive, blue being negative. (A) EC, (B) HP, (C) FC, (D) TC and (E) ADNI 


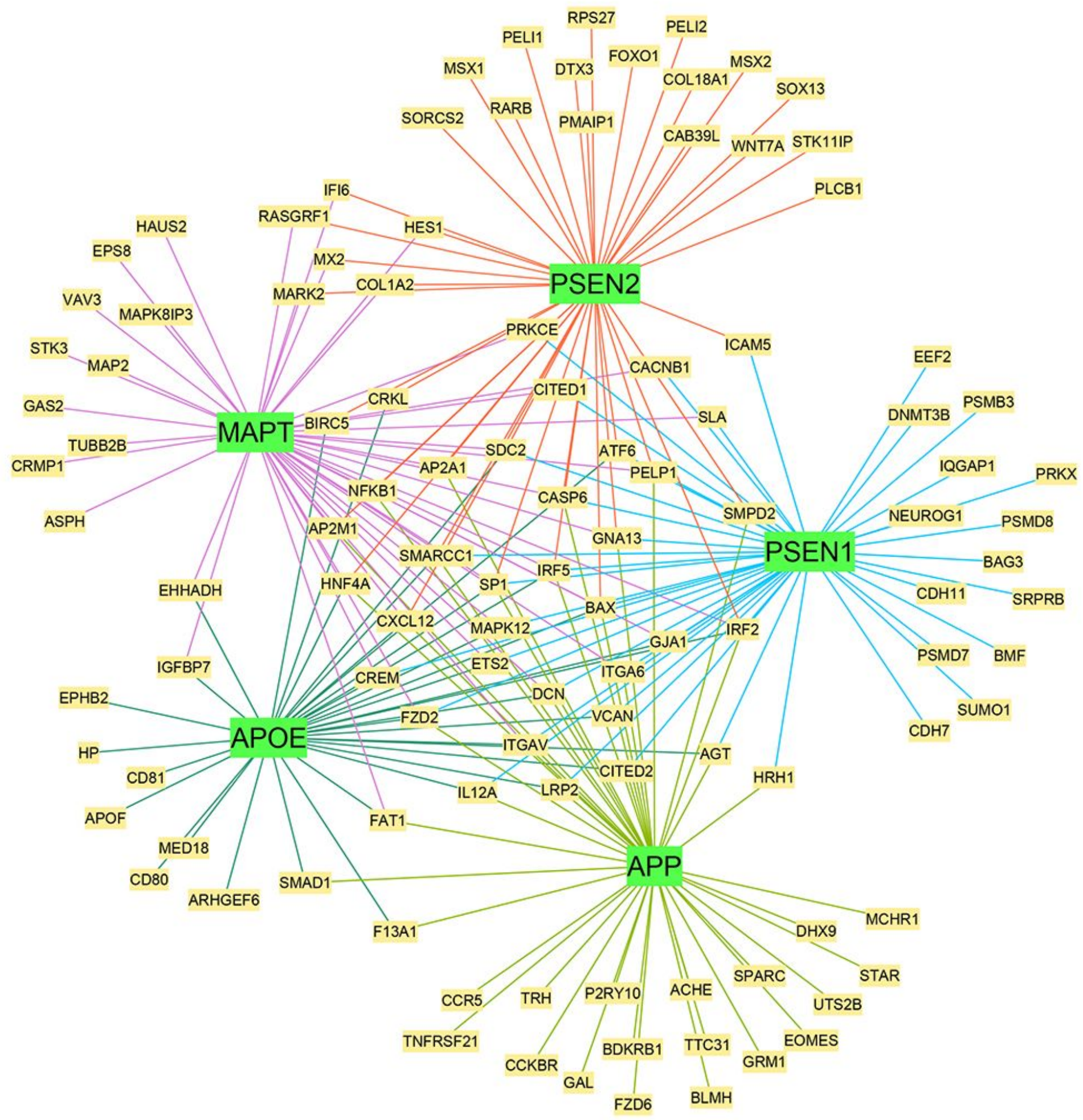

Figure 7

PPI network. The PPI network contained 5 well-known AD risk genes (green) and 209 (yellow) neighbors, which are from five AD-specific modules. 